\title{
What Exactly Is Shared Decision-Making? A Qualitative Study of Shared Decision-Making in Lung Cancer Screening
}

\author{
Anne C Melzer, MD MS ${ }^{1,2}$, Sara E. Golden, $M P H^{3}$, Sarah S. Ono, $P h D^{3,4}$, \\ Santanu Datta, $P h D, M B A^{5}$, Kristina Crothers, $M D^{6,7}$, and Christopher $G$. Slatore, \\ $M D, M S^{3,8,9}$
}

\begin{abstract}
${ }^{1}$ Center for Care Delivery and Outcomes Research, Minneapolis VA Healthcare System, Minneapolis, MN, USA; ${ }^{2}$ Division of Pulmonary and Critical Care, Department of Medicine, University of Minnesota, Minneapolis, MN, USA; ${ }^{3}$ Center to Improve Veteran Involvement in Care, VA Portland Health Care System, Portland, OR, USA; ${ }^{4}$ Department of Family Medicine, Oregon Health \& Science University, Portland, OR, USA; 5 Division of General Internal Medicine, Department of Medicine, Duke University, Durham, NC, USA; ${ }^{6}$ Section of Pulmonary, Critical Care and Sleep Medicine, VA Puget Sound Health Care System, Seattle, WA, USA; ${ }^{7}$ Division of Pulmonary, Critical Care and Sleep Medicine, Department of Medicine, University of Washington, Seattle, WA, USA; ${ }^{8}$ Division of Pulmonary \& Critical Care Medicine, Department of Medicine, Oregon Health \& Science University, Portland, OR, USA; ${ }^{9}$ Section of Pulmonary \& Critical Care Medicine, VA Portland Health Care System, Portland, OR, USA.
\end{abstract}

BACKGROUND: Shared decision-making (SDM) is widely recommended and required by the Centers for Medicare and Medicaid for patients considering lung cancer screening (LCS).

OBJECTIVE: We examined clinicians' communication practices and perceived barriers of SDM for LCS at three medical centers with established screening programs.

DESIGN: Multicenter qualitative study of clinicians participating in LCS.

APPROACH: We performed semi-structured interviews, which were transcribed and analyzed using directed content analysis, guided by a theoretical model of patientclinician communication.

PARTICIPANTS: We interviewed 24 clinicians including LCS coordinators (2), pulmonologists (3), and primary care providers (17), 4 of whom worked for the LCS program, a thoracic surgeon, and a radiologist.

RESULTS: All clinicians agreed with the goal of SDM, to ensure the screening decision was congruent with the patient's values. The depth and type of information presented by each clinician role varied considerably. LCS coordinators presented detailed information including numeric estimates of benefit and harm. Most PCPs explained the process more generally, focusing on logistics and the high rate of nodule detection. No clinician explicitly elicited values or communication preferences. Many PCPs tailored the conversation based on their implicit understanding of patients' values and preferences, gained from past experiences. PCPs reported that time, lack of detailed personal knowledge of LCS, and patient preferences were barriers to SDM. Many clinicians perceived that a significant proportion of patients were not interested in specific percentages and preferred to receive a clinician recommendation.

Electronic supplementary material The online version of this article (https://doi.org/10.1007/s11606-019-05516-3) contains supplementary material. which is available to authorized users.

Received March 1, 2019

Revised August 13, 2019

Accepted October 2, 2019

Published online November 19, 2019
CONCLUSIONS: Our results suggest that clinicians support the goal of SDM for LCS decisions but PCPs may not perform some of its elements. The lack of completion of some elements, such as PCPs' lack of in-depth information exchange, may reflect perceived patient preferences for communication. As LCS is implemented, further research is needed to support a personalized, patientcentered approach to produce better outcomes.

KEY WORDS: lung cancer screening; shared decision-making; communication.

$\mathrm{J}$ Gen Intern Med 35(2):546-53

DOI: $10.1007 / \mathrm{s} 11606-019-05516-3$

(1) Society of General Internal Medicine (This is a U.S. government work and not under copyright protection in the U.S.; foreign copyright protection may apply) 2019

\section{BACKGROUND}

Though the National Lung Screening Trial (NLST) identified clear benefits from lung cancer screening (LCS), ${ }^{1,2,4,5}$ it also identified a number of potential harms, including frequent identification of "false positives," incidental findings, and the potential for distress. ${ }^{4,6,7}$ Therefore, although the Centers for Medicare and Medicaid (CMS) agreed to cover LCS, ${ }^{8}$ for the first time, it mandated additional requirements that must be met in order to have the test reimbursed. ${ }^{8}$ One major requirement ${ }^{9}$ is that all patients receive information about the benefits and harms of LCS with a qualified healthcare provider prior to the low-dose CT scan (LDCT), commonly referred to as a "shared decision-making visit."

Patient-centered communication (PCC) is communication that is "closely congruent with, and responsive to, patients' wants, needs and preferences." 10 Shared decision-making (SDM) is one component of PCC ${ }^{11}$ in which patients and clinicians exchange information about patient values and preferences ("choice talk") and risks and benefits ("option talk") to make a decision in the way the patient prefers ("decision talk") (Fig. 1). ${ }^{12,13}$ CMS prioritizes information exchange and 


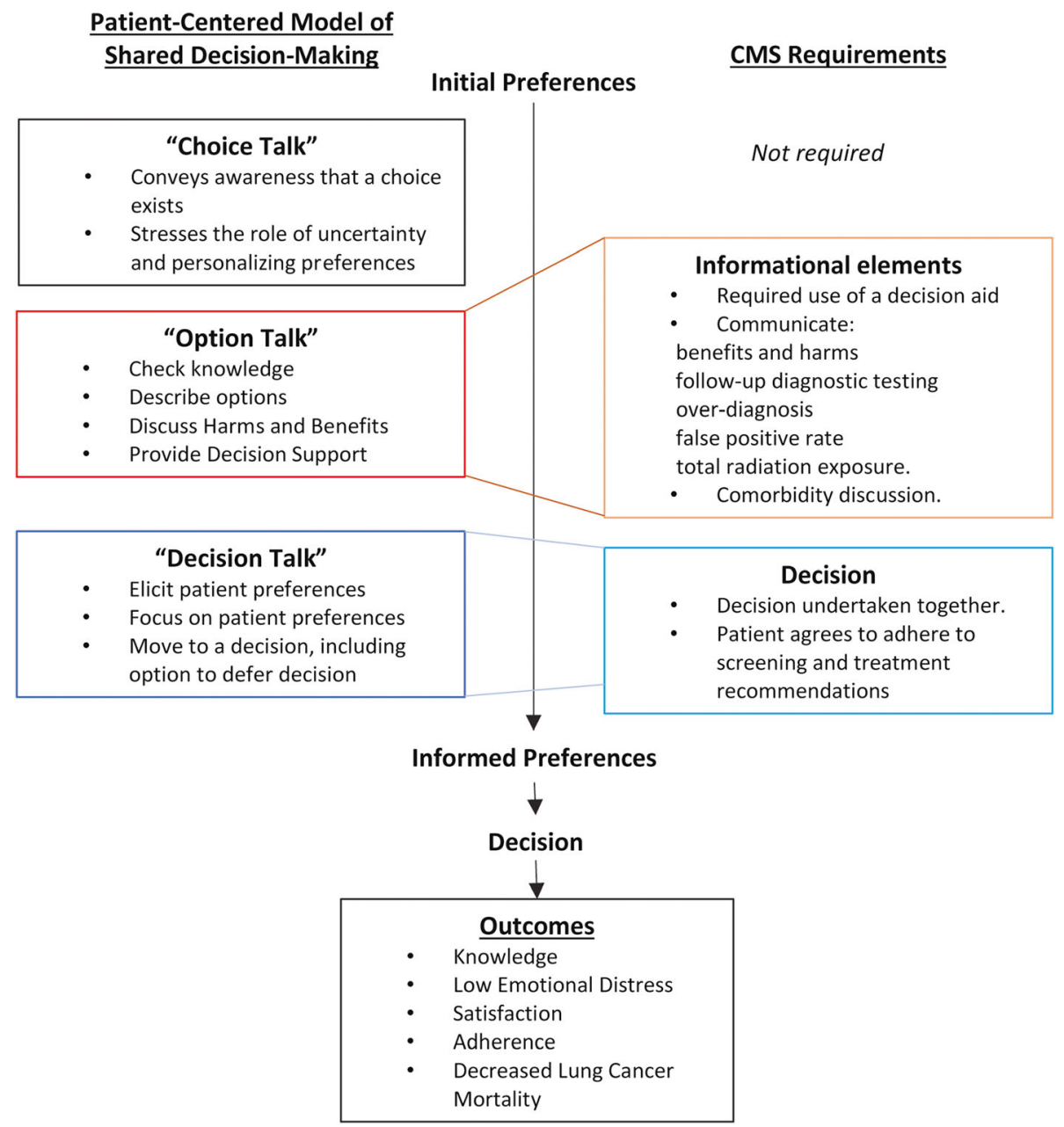

Figure 1 Comparison of patient-centered shared decision-making and Centers for Medicare and Medicaid (CMS) requirements for lung cancer screening (LCS). Based on the Elwyn model of shared decision-making.

defines an LCS SDM visit as including determination of eligibility, information about benefits and harms of screening including use of formal decision aids, counseling on the importance of adherence to screening, and counseling on tobacco abstinence, resulting in a shared decision by the clinician and patient together. However, this mandated process may prioritize one communication component, information exchange, over other domains such as consideration of patients values and preferences and thus may not in fact represent high-quality PCC.

Clinicians may not be performing LCS SDM visits in accordance with either CMS recommendations or recognized models of PCC. ${ }^{14-17}$ Based on a theoretical model of PCC, ${ }^{11}$ we sought to examine current communication practices and barriers to SDM for LCS at three medical centers with wellestablished LCS programs.

\section{METHODS}

We conducted a prospective, qualitative study to evaluate clinician views on SDM and PCC in the context of LCS. We recruited clinicians from three medical centers with established LCS programs: VA Portland Health Care System (VAPORHCS), Portland, OR; Minneapolis VA Medical Center, Minneapolis, MN; Duke University Medical Center, Durham, NC. A detailed description of the study has been published previously. ${ }^{3}$

\section{Sample}

We purposively recruited English-speaking clinicians who participated in LCS in diverse clinician roles, including LCS coordinators (LCS-Cs), pulmonologists (Pulm), a radiologist, a thoracic surgeon, and both physician and non-physician primary care providers (PCPs), recruiting until saturation of main themes. ${ }^{18}$

The study was IRB-approved at each site (VAPORHCS \#3482; Minneapolis VA \#4645-B; Duke \#Pro00073394).

LCS sites varied in care processes from highly centralized (all patients referred from PCPs for additional SDM, LDCT ordering conducted by the LCS-C), primarily driven by primary care (several PCPs served as program contacts, SDM, and ordering by individual providers), and another that used a shared process between primary care and the LCS program with central oversight. Though each center had offered 
education in LCS (e.g., presentations on the NLST, eligibility, and local process), none had mandated formal training for clinicians in conducting SDM. Coordinators received on-thejob training in completion of SDM visits. All clinicians are referred to as "she" to maintain anonymity. Numbers indicate individual clinicians.

\section{Procedures}

A semi-structured interview guide was used for consistency. Clinicians were asked to reflect on performing SDM, but were not explicitly asked their opinion of the CMS mandate. Participants were interviewed by phone and in-person based on location. All interviews were conducted by a single qualitative analyst (SEG) in a private space. The PI (CGS) did not perform interviews to avoid moderator response bias. Interviews were recorded, transcribed, deidentified, then audited for accuracy. Participants self-reported demographic and practice characteristics (Table 1).

\section{Analysis}

We used directed content analysis ${ }^{19}$ and ATLAS.ti 7.1.7 (ATLAS.ti Scientific Software Development GmbH) to organize and support analysis of qualitative data. The PI (CGS) and qualitative analyst (SEG) reviewed the first three completed transcripts to develop a preliminary codebook, refined, and

Table 1 Self-Reported Characteristics among Participants in Qualitative Interviews, $n=\mathbf{2 4}$

\begin{tabular}{ll}
\hline \hline Characteristic & N (\%)* or mean (SD) \\
\hline Age (year) & $42(12.5)$ \\
Gender, $n(\%)$ & $8(33 \%)$ \\
Male & $16(67 \%)$ \\
Female & $18(75 \%)$ \\
Race, $n(\%)$ & $3(13 \%)$ \\
Caucasian & $3(13 \%)$ \\
Asian & \\
Black/African American & $10(42 \%)$ \\
Specialty, $n(\%)$ & $3(12 \%)$ \\
Primary care physician/general internist & $1(4 \%)$ \\
Pulmonologist & $1(4 \%)$ \\
Thoracic Surgeon & $4(17 \%)$ \\
Radiologist & $5(21 \%)$ \\
Physician assistant & \\
Nurse practitioner/registered nurse & $14(58 \%)$ \\
Years in practice (since end of training), $n(\%)$ & $5(21 \%)$ \\
$0-10$ & $5(21 \%)$ \\
$11-20$ & $8(33 \%)$ \\
$>21$ & $8(33 \%)$ \\
Site, $n(\%)$ & $8(33 \%)$ \\
Portland & \\
Minneapolis & $14(58 \%)$ \\
Durham & $4(17 \%)$ \\
Years spent in current site, $n(\%)$ & $6(25)$ \\
$0-5$ & $16(67 \%)$ \\
6-10 & $8(33 \%)$ \\
> 11 & $16(67 \%)$ \\
Practice setting, $n(\%)$ & $4(17 \%)$ \\
Government & $4(17 \%)$ \\
University based & \\
Type of clinic, $n(\%)$ & \\
Internal medicine/primary care & \\
Pulmonary & \\
Other & \\
\hline
\end{tabular}

*Percentages are of non-missing data revised twice by consensus (SEG, CGS, LM, SSO). After changes to the codebook, initial transcripts were recoded. We utilized an audit trail to track any modifications made to the codebook and analysis. The remaining transcripts were coded by two analysts (SEG, LM), with another experienced qualitative coder (SSO) reviewing every 5 transcripts to ensure appropriate intercoder reliability. Throughout this process, we evaluated overlapping coding and uncoded text to verify appropriateness and completeness of the codebook. Coded transcripts were reviewed again after coding by an additional reviewer $(\mathrm{ACM})$ to ensure agreement with the identified codes.

\section{RESULTS}

Our sample included two LCS-Cs, three pulmonologists, seventeen PCPs, one thoracic surgeon, and one radiologist. The four PCPs who served as site contacts for their LCS program are referred to throughout as PCPs, as their responses most resembled other PCPs. PCPs represented a mixture of provider types (physicians, nurse practitioners, physician assistants) and practice settings, including general primary care and tobacco cessation clinics. The table presents self-reported demographic characteristics. We identified themes related to three major aspects of PCC: information exchange, SDM, and patient reactions. Additional supporting quotations are available in the online supplement.

\section{Information Exchange}

The exchange of information that took place during LCS decision-making encounters varied by clinician role, particularly in the level of detail provided.

Depth and Type of Information. All clinicians agreed that "adequate" information was a necessary part of the process and that this information should be provided at the patient's level of health literacy. Judgments of health literacy were implicit and based on past and current clinical encounters. The depth and type of information considered adequate varied by provider type.

LCS-Cs reported extensive discussions with patients regarding the magnitude of benefit and the frequency of common risks, including percentages and printed and electronic decision aids, which included pictorial representations of screening risks and benefits. Coordinators reported addressing concepts like overdiagnosis, false positives, and the relatively small margin of benefit. Despite providing these details, one coordinator felt that this information often "goes over their heads," but still felt it was important to provide, as "they don't know what they don't know." Pulmonologists endorsed close relationships with the LCS-C and were comfortable providing general information (confirming eligibility, describing LDCT, annual screens) and referring patients to the coordinator for a more detailed conversation. Pulm 2 felt that patients understood the concept of overdiagnosis - the possibility of 
diagnosing a cancer that would not have been clinically significant — quite well. Pulm 22 suggested that LCS information could be "pared down," for example not discussing in detail relatively rare downstream consequences such as invasive testing for non-cancer nodules. However, many pieces (e.g., need for adherence, frequent false positives) were essential. The radiologist noted that patients coming through the program seemed to have "a lot" of education about LCS and appeared well versed in the process and that overall the transitions in care seemed "seamless." Both the radiologist and the surgeon indicated strong support of the program, describing themselves as "believers" due to the many cancers that had been found, treated, and evaluated through the process of multidisciplinary review.

Nearly all PCPs reported providing more generalized information about LCS, focusing on logistical aspects of screening (e.g., annual scan, possible copays, "simple" CT scan) rather than specific outcomes. Few used decision aids. Many felt that available decision aids, even the one used by their program, were excessive in length. Most PCPs did not provide numbers or percentages regarding benefits or harms, feeling that this information is potentially confusing, not necessary for patients to make a screening decision, and not a helpful way to communicate for most patients. Some noted "I don't use numbers with my patients" (PCP 25). In general, PCPs did not perceive LCS as a high-risk intervention, sometimes referring to it as "just a CT scan." Only one indicated "there's a lot of risks actually involved in screening (PCP 17)." Multiple providers stated that they purposely limited the amount of information presented. They felt that discussing overdiagnosis or downstream consequences of false positives was "way too much information" (PCP 13). They perceived that providing excessive information could cause the patient to disengage and, in effect, be similar to giving them "no information at all" (PCP 25).

Multiple PCPs stated that most patients were unaware of LCS but that it was easily put into context with familiar screening tests. Clinicians indicated that few patients asked any questions at all. Occasional questions pertained primarily to logistics or radiation exposure. In those instances, the PCP would either address it herself or refer the patient to the LCS-C. Clinicians felt the lack of questions demonstrated that patients did not desire more information beyond what they had received.

The benefits of screening were framed variably, from finding cancer earlier to helping patients "live longer" (PCP 18/ 19). Several PCPs suggested that better "one-pagers"-described as very brief decision aids summarizing eligibility criteria and annual LDCT - would be helpful for both patients and providers. LCS-Cs and pulmonologists noted that patients referred for screening received "really variable information" (Pulm 22) from their PCP. They also felt that PCPs had little expertise in LCS; therefore, SDM was better performed by program staff.

Tailoring of Information. All clinicians agreed that some degree of tailoring of information was necessary but interpreted the idea of tailoring in different ways. These interpretations included the following: providing tailored risk estimates, tailoring the level of detail to the patient's health literacy, and tailoring the discussion to a patient's baseline level of anxiety. The judgments that contributed to how clinicians tailored the communication were implicit; no clinician indicated that they ask a patient how much or what kind of information they preferred.

LCS-Cs provided comprehensive education to everyone who was willing to accept it, but with different levels of detail and pace of new concepts depending on perceived health literacy. Coordinator 5 discussed overdiagnosis more thoroughly with older, sicker patients than with younger ones and used a web-based decision aid with personalized cancer risk estimates to guide screening discussions. Pulmonologist 12 tailored the conversation based on the patient's initial reaction to screening (i.e., for or against), whether they are "data people," or whether they "just want a recommendation."

Many PCPs endorsed a similar process, providing more detail for patients who present as being data driven or anxious about the process. PCP 14 noted, "It's usually obvious who really wants to talk and who doesn't," and PCP 24 noted that few patients want to address all the "ifs and possible outcomes." Though no PCPs directly assessed communication preferences, many indicated that conversations were tailored based on knowledge of the patient over time from previous clinical encounters. Pre-existing mental health comorbidities and health literacy were key factors in determining the type of information and level of detail offered. Only one PCP endorsed a different approach, indicating that regardless of patient background she provided everyone with "the same spiel" (PCP 23).

\section{Shared Decision-Making}

All clinician types were aware of the need for SDM and identified barriers to and inconsistencies in conducting SDM. Patient Values. Clinicians of all roles agreed that ensuring a screening decision in line with a patient's values is key. No clinicians mentioned eliciting values explicitly. Many PCPs indicated that past experiences with a patient guided their communication the most, allowing for a more streamlined discussion. This process was described as "gaug(ing) where they're at" (PCP 25) to inform a recommendation. The process was noted to be more difficult when a patient was new, such as during a consultation in a tobacco treatment clinic. PCPs noted they could usually predict how a patient would receive the opportunity to be screened based on prior knowledge of their fears about testing and past screening decisions.

Coordinators stressed that the purpose of SDM was to ensure that each patient felt that the risk/benefit ratio was appropriate to him/her personally and that the decision therefore could not come solely from a provider. Although they stressed the personal nature of the decision, they did not indicate how they assessed values. They saw their role as highly education-focused, giving patients knowledge of LCS 
to make their own decision, working within the CMS requirements. However, coordinator 1 stressed that knowledge, while important, did not seem to be the key deciding factor, but that patients' pre-existing views of screening tests was more important.

Barriers to Shared Decision-Making. Providers in all roles indicated that time was a significant issue, as SDM was perceived to be a lengthy process. Lack of time led to lower priority for completion. PCPs estimated a "true" screening discussion took between 5 and $10 \mathrm{~min}$, which was viewed as not feasible in a 15-20-min visit. LCS-Cs had more time for discussions. Clinicians of varied roles noted that, while SDM was explicitly required for LCS, the necessary components and how this differed from the usual method of making medical decisions were not clear. Several suggested that more support to identify eligible patients and access electronic patient information would be helpful.

Lack of patient engagement in the process of decisionmaking was a barrier identified by all clinician types. Many patients were perceived as being uninterested in details and terminated the discussion. Both PCPs and pulmonologists reported that a large number of patients, particularly older patients, requested a firm recommendation. These patients appeared to base their decision entirely on this recommendation. In contrast, rare patients wanted to make all decisions themselves after a detailed discussion, or came to the discussion wanting to be screened. Coordinator 5 indicated that the local process, in which a PCP refers a patient to the program to complete SDM, was a significant barrier to adequate information exchange, as patients interpreted the referral as an order for LCS. Their "mind was made up" to get the LDCT regardless of what the coordinator said. Attempting to have a more detailed conversation appeared to be against the preferences of the patient. Coordinator 5 noted that she "hates not educating people" but did not press disinterested patients to participate in further SDM.

Patient Choice and Provider Influence. All clinicians indicated that the screening decision rests with the patient and felt they made it clear that screening was optional. However, as above, some clinicians noted a perception that many patients "don't want a choice." PCPs indicated a tension between ensuring the patient's ability to choose and feeling that it is appropriate, and often explicitly requested, to give a firm recommendation. Many indicated that providing a recommendation, and having it followed, is a fundamental part of a high-quality patient-PCP relationship. Coordinator 1 expressly strove to be "neutral" towards screening when performing SDM. She noted that the resulting SDM discussions caused patients to change their minds to both undergo or forgo screening in relation to their initial preferences. She viewed this as a positive result of SDM as it showed patients were processing information and changing their decision from their initial reaction.

Despite PCPs indicating that LCS is offered as a choice, LCS-Cs felt that many patients did not perceive it that way and felt obligated to follow their PCP's recommendation. LCS-Cs and pulmonologists noted that many patients would accept screening during the visit with the PCP, but decline when subsequently offered it by the coordinator or scheduler, or simply never answer the phone or show up for the test. This lack of follow-through led them to question how strongly PCPs are influencing patients' decisions in the moment. LCS-Cs were unsure whether this disconnect was due to strong recommendations on the part of PCPs or to a "white coat effect" of simply agreeing with doctors.

\section{Perceived Patient Reactions: Screening Decision and Anxiety}

All but one PCP reported that nearly all patients accept screening readily when offered. PCPs and pulmonologists noted "[patients] just agree pretty much...100\% of the time," (PCP $8)$. Only one PCP had more patients decline than accept the offer, with acceptance rates of "80-20 against" (PCP 20), which she attributed to a very frank discussion of risks and benefits in the setting of competing medical issues. PCPs felt that "decliners" were in two groups: decliners of screening tests in general and those who felt anxious or "(didn't want) to know" about lung cancer. Patients who declined all screening tests gave several reasons, including comorbidities, cost, or a belief that cancer "won't happen to them" (PCP 26). Patients who declined LCS specifically cited anxiety over waiting long periods of time to know if a nodule was benign. Although PCPs indicated that anxiety was a common reason to decline, declining was rare and very few patients appeared anxious about undergoing LCS. Clinicians noted that few patients were anxious or distressed about the possibility of small nodules ("false positives") or potentially invasive procedures.

Coordinators reported different experiences based on their program structure. One found that significant numbers of patients declined at the time of her SDM discussion. The other noted this was rare among patients she spoke with, as patients who initially declined LCS never had a referral placed to her to discuss screening. Both noted that many patients never answered the phone to make an appointment for SDM, never scheduled the test, or never showed up. Both coordinators felt that patients who did not follow through on screening were making a decision "with their feet."

\section{DISCUSSION}

In this multicenter, qualitative study of clinicians with different roles in LCS processes, we found variable decisionmaking communication practices. Overall, LCS-Cs and some PCPs completed most aspects of the CMS mandated process, with the exception of using printed decision aids. Despite this, 
many aspects of PCC and SDM were not reported to be reliably occurring. By using the term "shared decision-making," the CMS requirements imply the need to assess communication and decision-making preferences. However, CMS does not specify how these critical aspects of SDM should occur and instead focuses on information exchange ("option talk"). No clinician expressly assessed communication preferences or preferences for role in decision-making, or elicited patient values - key parts of "choice talk" and "decision talk" - though many PCPs felt they had a good implicit understanding of these characteristics of their patients. Unfortunately, this lack of assessment of patient preferences is in line with other studies of SDM for LCS. ${ }^{16}$ The informationcentered SDM process currently occurring may not lead to decisions reflective of patient preferences.

Many models of medical decision-making do not account for the influence of the existing therapeutic relationship on the decision-making process. Some SDM models, including one endorsed by the US Preventative Services Task Force, include clinician recommendations as a part of SDM, ${ }^{9,20}$ provided that receiving a recommendation reflects patient preferences. PCPs articulated that elements of SDM did not occur during a single discrete visit, but took place over time as the provider came to know the patient's values and preferences. However, in the absence of formally assessing a patient's values and preferences, it is unclear whether the implicit understanding reported by PCPs accurately reflects patient preferences. Though patients do vary in their communication preferences, prior research suggests that implicit assessments of communication preferences by clinicians are often inaccurate. ${ }^{21,22}$ Explicitly assessing the communication and decision-making preferences of patients considering LCS may result in improved patient-centered outcomes and decision quality.

The sampled clinicians noted many barriers to SDM, though notably they did not report that SDM is harmful or unhelpful. Similar to prior studies, the time required to complete SDM was a commonly cited barrier. ${ }^{23,24}$ The time and complexity of the SDM process may be an impediment to the adoption of LCS in the community, ${ }^{25}$ perhaps contributing to low observed rates of screening. ${ }^{26,27}$ Though time was a barrier, clinicians reported other reasons for not completing all components of the CMS mandated process. These reasons included a perception that patients do not want, need, or understand extensive information on risks and benefits, that decision aids are excessively detailed, and that many patients prefer firm clinician recommendations. The beliefs described by our sample are at odds with the process of SDM as described in several commonly cited models ${ }^{13,28,29}$ and the CMS process, yet may accurately reflect the preferences of many patients. Although most patients prefer SDM, some prefer a passive role, as reported by our clinicians. ${ }^{30,31}$ Studies support a variety of patient communication preferences such that a one-size-fits-all approach to decision-making is likely to result in low-quality communication for a subset of patients.
Up to half of patients prefer their physicians make the final decision. ${ }^{32}$ Other populations of patients indicate that they do want detailed information, are open to the use of decision aids, and prefer an active role in making health decisions. ${ }^{33,34}$ The current list of CMS requirements for the SDM visit may conflict with the preferences of many patients. Despite the importance of SDM to both LCS and other medical decisions, most clinicians receive little formal training. ${ }^{35,36}$ Our findings are similar to other studies of SDM for both cancer and lung nodule care. Clinicians endorse that SDM is central to the way they communicate with patients, but are often unaware or unsupportive of specific elements. ${ }^{37-39}$ For example, many worry that patients perceive the lack of a recommendation as a lack of knowledge or confidence, which may erode trust. ${ }^{40}$ In contrast, patients who are included in their medical decisions report higher quality communication, suggesting that this perception is false. ${ }^{41-43}$

Although a separate SDM encounter with a coordinator seems like a solution to the time constraints and lack of detailed screening knowledge noted by PCPs, LCS-Cs indicated that this did not necessarily result in an ideal context for SDM. Coordinators adhered most closely to the CMS requirements, but still reported issues with completing SDM. They reported that many patients felt the decision had already been made at the time of referral and the discussion was perceived as a formality. Coordinators have no existing relationship with referred patients, which may hamper the decision-making process. Though many patients accepted the offer from their PCP, a large proportion never replied to further attempts at care coordination. This lack of response may reflect their "true" screening preference or patients deciding that the obstacles to getting the LDCT outweigh the perceived benefits. Similar to previous studies, of those who actually participated in an encounter with the LCS coordinator, nearly all elected to be screened. ${ }^{44}$ Patients who present for a discussion with an LCS program may be enthusiastic adopters, which may cut down on no-shows and nonadherence. As facilities design their programs, determining who will undertake the full SDM discussion is an important aspect, as this likely determines which patients will decline at different points in the process, with an unclear impact on who ultimately undergoes an LDCT. Telehealth visits may allow patients to discuss LCS with a trained coordinator without the barrier of distance.

Study limitations included that aspects of communication were based on self-report and therefore may not fully reflect the communication that took place with patients. All clinicians were located at institutions with well-established screening programs, and their responses may not be generalizable to institutionslacking a formal program. Though we sampled diverse roles, we may not have reached saturation - the point at which no new concepts are generated - forall concepts for all roles. Our primarily white sample does not allow us to comment on racial variation in SDM. The lower sample size associated with a qualitative study may not generalize to all clinicians. We will report on patients' perceptions of LCS decision-making but decided those analyses were outside the scope of a single paper. ${ }^{3}$ 


\section{CONCLUSION}

The process of decision-making for LCS has been recommended to include a more extensive list of requirements than for other cancer screening tests, despite a lack of data supporting this approach. Even at centers with well-established screening programs, few clinicians report providing "true" SDM or fully complying with CMS requirements. Though some of the limitations on providing SDM were related to barriers such as time constraints and lack of detailed knowledge of LCS, many PCPs reported a fundamental disagreement with a focus on information exchange, feeling that the communication and SDM should be more tailored to the patient-clinician relationship. As LCS is widely implemented, processes of care that ensure that patients have access to their preferred mode of communication and decision-making may produce better outcomes than processes that depend on a "check-box" approach.

Corresponding Author: Anne C Melzer, MD MS; Center for Care Delivery and Outcomes Research, Minneapolis VA Healthcare System. Minneapolis, MN, USA (e-mail: Anne.melzer2@va.gov).

Funding Information This project was funded by a grant from the American Cancer Society (RSG-15-155-01, Slatore (PI)). The study was supported by resources from the VA Portland Health Care System, Portland, OR, and the Minneapolis VA Health Care System, Minneapolis, $M N$.

\section{Compliance with Ethical Standards:}

The study was IRB-approved at each site (VAPORHCS \#3482; Minneapolis VA \#4645-B; Duke \#Pro00073394).

Conflict of Interest: The authors have no financial conflicts of interest to declare. ACM, KC, and CGS are medical directors of lung cancer screening programs at the institutions where they are employed but do not receive additional compensation for these roles.

Disclaimer: The Department of Veterans Affairs did not have a role in the conduct of the study, in the collection, management, analysis, interpretation of data, or in the preparation of the manuscript. The views expressed in this article are those of the authors and do not necessarily represent the views of the Department of Veterans Affairs or the U.S. Government.

\section{REFERENCES}

1. Moyer VA. Screening for lung cancer: U.S. Preventive Services Task Force recommendation statement. Ann Intern Med. 2014;160:330-338.

2. Mazzone P, Powell CA, Arenberg D, et al. Components necessary for high-quality lung cancer screening: American college of chest physicians and american thoracic society policy statement. Chest. 2015;147:295303.

3. Miranda LS, Datta S, Melzer AC, et al. Rationale and Design of the Lung Cancer Screening Implementation. Evaluation of Patient-Centered Care Study. Ann Am Thorac Soc. 2017;14:1581-1590.

4. Aberle DR, Adams AM, Berg CD, et al. Reduced lung-cancer mortality with low-dose computed tomographic screening. N Engl J Med. 2011;365:395-409.

5. Wood DE. National Comprehensive Cancer Network (NCCN) Clinical Practice Guidelines for Lung Cancer Screening. Thoracic Surgery Clinics. 25:185-197.
6. Harris RP, Sheridan SL, Lewis CL, et al. The harms of screening: A proposed taxonomy and application to lung cancer screening. JAMA Intern Med. 2014;174:281-286.

7. Slatore CG, Sullivan DR, Pappas M, Humphrey LL. PatientCentered Outcomes among Lung Cancer Screening Recipients with Computed Tomography: A Systematic Review. J Thorac Oncol. 2014;9:927-934.

8. Centers for Medicare \& Medicaid Services. Decision memo for screening for lung cancer with low dose computed tomography (LDCT) (CAG-00439 N). Baltimore: Centers for Medicare and Medicaid, U.S. Department of Health and Human Services. (https://www.cms.gov/medicare-coveragedatabase/details/nca-decision-memo.aspx?NCAId $=274$. Accessed $\mathrm{Au}^{-}$ gust 30, 2019.)

9. Sheridan SL, Harris RP, Woolf SH, Force SD. Shared decision making about screening and chemoprevention: a suggested approach from the US Preventive Services Task Force. Am J Prev Med. 2004;26:56-66.

10. Laine C, Davidoff F. Patient-centered medicine. A professional evolution. JAMA. 1996;275(2):152-156.

11. Mead N, Bower P. Patient-centredness: a conceptual framework and review of the empirical literature. Soc Sci Med (1982). 2000;51:10871110 .

12. Elwyn G, Cochran N, Pignone M. Shared decision making-the importance of diagnosing preferences. JAMA Intern Med. 2017; 17:1239-1240.

13. Elwyn G, Frosch D, Thomson R, et al. Shared Decision Making: A Model for Clinical Practice. J Gen Intern Med. 2012;27:1361-1367.

14. Kanodra NM, Pope C, Halbert CH, Silvestri GA, Rice LJ, Tanner NT. Primary Care Provider and Patient Perspectives on Lung Cancer Screening. A Qualitative Study. Ann Am Thorac Soc. 2016;13:1977-1982.

15. Triplette M, Kross EK, Mann BA, et al. An Assessment of Primary Care and Pulmonary Provider Perspectives on Lung Cancer Screening. Ann Am Thorac Soc. 2018;15:69-75.

16. Brenner AT, Malo TL, Margolis M, et al. Evaluating Shared Decision Making for Lung Cancer Screening. JAMA Intern Med. 2018,178:13111316

17. Ersek JL, Eberth JM, McDonnell KK, et al. Knowledge of, attitudes toward, and use of low-dose computed tomography for lung cancer screening among family physicians. Cancer. 2016

18. Sandelowski M. Sample size in qualitative research. Res Nurs Health. 1995; 18(2): 179-183.

19. Hsieh HF, Shannon SE. Three approaches to qualitative content analysis. Qual Health Res. 2005; 15: 1277-1288.

20. Callon W, Beach MC, Links AR, Wasserman C, Boss EF. An expanded framework to define and measure shared decision-making in dialogue: A 'top-down' and 'bottom-up' approach. Patient Educ Couns. 2018;101:1368-1377.

21. Elkin EB, Kim SH, Casper ES, Kissane DW, Schrag D. Desire for information and involvement in treatment decisions: elderly cancer patients' preferences and their physicians' perceptions. J Clin Oncol. 2007;25:5275-5280.

22. Bruera E, Willey JS, Lynn Palmer J, Rosales M. Treatment decisions for breast carcinoma. Cancer. 2002;94:2076-2080.

23. Tai-Seale M, McGuire TG, Zhang W. Time allocation in primary care office visits. Health Serv Res. 2007;42:1871-1894.

24. Caverly TJ, Hayward RA, Burke JF. Much to do with nothing: microsimulation study on time management in primary care. BMJ. 2018,363:k4983.

25. Carter-Harris L, Gould MK. Multilevel Barriers to the Successful Implementation of Lung Cancer Screening: Why Does It Have to Be So Hard? Ann Am Thorac Soc. 2017;14:1261-1265.

26. Huo J, Shen C, Volk RJ, Shih Y-CT. Use of CT and Chest Radiography for Lung Cancer Screening Before and After Publication of Screening Guidelines: Intended and Unintended Uptake. JAMA Intern Med. 2017; 177:439-441.

27. Henderson LM, Jones LM, Marsh MW, Benefield T, Rivera MP, Molina PL. Lung Cancer Screening Practices in North Carolina CT Facilities. J Am Coll Radiol. 2017;14:166-170.

28. Charles C, Gafni A, Whelan T. Shared decision-making in the medical encounter: what does it mean? (or it takes at least two to tango). Social Sci Med (1982). 1997;44:681-692.

29. Murray E, Charles C, Gafni A. Shared decision-making in primary care: tailoring the Charles et al. model to fit the context of general practice. Patient Educ Couns. 2006;62:205-211.

30. Deber RB, Kraetschmer N, Urowitz S, Sharpe N. Do people want to be autonomous patients? Preferred roles in treatment decision-making in several patient populations. Health Expect. 2007;10:248-258. 
31. Kraetschmer N, Sharpe N, Urowitz S, Deber RB. How does trust affect patient preferences for participation in decision-making? Health Expect. 2004;7:317-326.

32. Levinson W, Kao A, Kuby A, Thisted RA. Not all patients want to participate in decision making. A national study of public preferences. J Gen Intern Med. 2005;20:531-535.

33. Crothers K, Kross E, Reisch LM, et al. Patients' Attitudes Regarding Lung Cancer Screening and Decision Aids: A Survey and Focus Group Study. Ann Am Thorac Soc. 2016;13:1992-2001

34. Wiener RS, Koppelman E, Bolton R, et al. Patient and Clinician Perspectives on Shared Decision-making in Early Adopting Lung Cancer Screening Programs: a Qualitative Study. J Gen Intern Med. 2018;33:1035-1042.

35. Legare F, Witteman HO. Shared decision making: examining key elements and barriers to adoption into routine clinical practice. Health Aff (Millwood). 2013;32:276-284.

36. Friedberg MW, Van Busum $\mathbf{K}$, Wexler R, Bowen M, Schneider EC. A Demonstration Of Shared Decision Making In Primary Care Highlights Barriers To Adoption And Potential Remedies. Health Affairs. 2013;32:268-275.

37. Golden SE, Wiener RS, Sullivan D, Ganzini L, Slatore CG. Primary Care Providers and a System Problem: A Qualitative Study of Clinicians Caring for Patients With Incidental Pulmonary Nodules. Chest. 2015; 148: 1422-1429.

38. Iaccarino JM, Simmons $\mathbf{J}$, Gould $\mathbf{M K}$, et al. Clinical Equipoise and Shared Decision-making in Pulmonary Nodule Management. A Survey of American Thoracic Society Clinicians. Ann Am Thorac Soc. 2017; 14:968975.
39. Rocque G, Miller-Sonnet E, Balch A, et al. Engaging Multidisciplinary Stakeholders to Drive Shared Decision-Making in Oncology. J Palliat Care. 2019;34:29-31.

40. Zeuner R, Frosch DL, Kuzemchak MD, Politi MC. Physicians' perceptions of shared decision-making behaviours: a qualitative study demonstrating the continued chasm between aspirations and clinical practice. Health Expect. 2015;18:2465-2476.

41. Golden SE, Thomas CR, Jr., Moghanaki D, Slatore CG. Dumping the information bucket: A qualitative study of clinicians caring for patients with early stage non-small cell lung cancer. Patient Educ Couns. 2017;100:861-870.

42. Lillie SE, Partin MR, Rice $\mathbf{K}$, et al. VA Evidence-based Synthesis Program Reports. The Effects of Shared Decision Making on Cancer Screening - A Systematic Review. Washington (DC): Department of Veterans Affairs (US); 2014.

43. Vogel BA, Leonhart R, Helmes AW. Communication matters: the impact of communication and participation in decision making on breast cancer patients' depression and quality of life. Patient Educ Couns.. 2009;77:391-397.

44. Mazzone PJ, Tenenbaum A, Seeley M, et al. Impact of a Lung Cancer Screening Counseling and Shared Decision-Making Visit. Chest. 2017;151:572-578.

Publisher's Note Springer Nature remains neutral with regard to jurisdictional claims in published maps and institutional affiliations. 